\title{
VARIABILIDAD ESTACIONAL DE LOS CAUDALES EN ECUADOR EN EL PERIODO 1964-2014
}

\author{
C. QUISHPE-VÁZQUEZ, M. GARCÍA-VALDECASAS-OJEDA, \\ S.R. GÁMIZ-FORTIS, Y. CASTRO-DÍEZ and M.J. ESTEBAN-PARRA \\ Applied Physics Department, University of Granada, Granada, Spain. \\ cesarqv@correo.ugr.es
}

\section{RESUMEN}

El objetivo principal de este estudio es analizar las tendencias en el caudal de los ríos de Ecuador. Para este fin, se han utilizado los datos de caudal de la red de estaciones hidrológicas, proporcionados por el Instituto Nacional de Meteorología e Hidrología de Ecuador (IHNAMI), distribuidas en el territorio ecuatoriano y situadas estratégicamente en las cuencas de los principales ríos. Se seleccionó un número de 26 estaciones con disponibilidad de datos diarios sin discontinuidad durante el período de 51 años (1964-2014). Mediante el test de Man-Kendall y pendiente de Sen, se han evaluado las tendencias del caudal a escala mensual. Por último, se analiza la relación entre la variabilidad del caudal y el ENSO, fuente principal de variabilidad climática en la región.

Palabras clave: Ecuador, caudal, tendencias, variabilidad estacional.

\section{ASTRACT}

The main objective of this study is to analyze trends in the streamflow of the rivers of Ecuador. For this end, we use the streamflow data from the network of hydrological stations, provided by the Instituto Nacional de Meteorología e Hidrología de Ecuador (IHNAMI), distributed over the Ecuadorian territory and strategically located in the watersheds of its main rivers. A number of 26 stations with a continuous period of daily data during 51 years period (1964-2014) was selected. Trends of the streamflow rates have been evaluated at monthly time scales using Man-Kendall test and Sens slope. Finally, we analyze the relationship between the streamflow variability and the ENSO, which is the major climate variability source in the region.

Key words: Ecuador, streamflow, trends, seasonal variability.

\section{INTRODUCCIÓN}

La comprensión de la dinámica de caudales constituye uno de los temas más importantes en Hidrología y en cuestiones relativas al estudio de los recursos hídricos, ya que es un asunto de relevancia para la correcta gestión de estos. La dinámica de caudales se rige por diversos mecanismos físicos que actúan sobre una amplia gama de escalas temporales y espaciales. Por ejemplo, el caudal no sólo depende de la distribución de la precipitación en el tiempo y en el espacio, sino también del tipo y estado de la cuenca, que a su vez depende de las condiciones climáticas, el estado de la vegetación, etc. Además, casi todos los mecanismos que intervienen en 
el proceso de generación de escorrentía superficial presentan algún grado de no linealidad. Todos estos factores hacen que la modelización de caudales sea compleja. Sin embargo, en la última década, ha habido una considerable mejora en la capacidad de describir y modelar sistemas complejos como puede ser una cuenca fluvial. Los resultados de estos estudios son alentadores, ya que se consiguen razonablemente buenas predicciones de variables hidrológicas, en particular, del caudal (Jayawardena and Gurung, 1994; Lisi and Villi, 2001).

El caudal de un río puede ser una variable útil para conocer la disponibilidad de agua dulce. Además, puede ser utilizado como un factor para evaluar posibles cambios en el clima, que se han producido en los últimos años en una determinada zona. Así, en el presente estudio se analizan las tendencias de los caudales en los ríos de Ecuador, explorando la posibilidad de utilizar este indicador como una variable de predicción del clima a escala mensual y estacional, así como buscando una relación entre los eventos ENSO y las precipitaciones en la región.

\subsection{Zona de estudio}

Los Andes tropicales es, hidrológicamente, una de las más diversas regiones del mundo. La convergencia de varios sistemas climáticos, combinada con la topografía empinada, da lugar a gradientes de hidroclimáticos muy marcados. La precipitación supera fácilmente los $5000 \mathrm{~mm}$ en las laderas de la cuenca del Amazonas y en la región del Chocó de Colombia, mientras que muchas regiones en el valle interandino tienen un clima semiárido o árido (Vuille et al., 2000; Buytaert et al., 2006). Del mismo modo, los gradientes de tipos de suelo y la vegetación son impulsados por los gradientes de temperatura y precipitación, así como la variabilidad geológica, la actividad volcánica y la interferencia humana (Buytaert et al., 2006). Estos gradientes dan lugar a una rica variedad de sistemas hidrológicos con un comportamiento muy diferente.

Debido a la presencia de la cordillera de los Andes y a la influencia del mar, el Ecuador continental se halla climatológicamente fragmentado en diversos sectores. Además, a causa de su ubicación ecuatorial, cada zona climática presenta solo dos estaciones definidas: la húmeda y la seca. Para el presente estudio se ha definido la estación húmeda como aquella que va desde enero a junio, y la estación seca desde julio hasta diciembre. Debido a sus emplazamientos próximos a la línea ecuatorial, no ocurren verdaderos inviernos y veranos.

El Ecuador es un país rico en recursos hídricos. Según el Consejo Nacional de Recursos Hídricos (http://cice.org.ec/cnrh/cnrh.htm), existe a nivel nacional una escorrentía media total de $432.000 \mathrm{hm}^{3} / \mathrm{año}$, con una escorrentía específica de $1600 \mathrm{~mm} / \mathrm{año}$, muy superior a la media mundial que es del orden de $300 \mathrm{~mm} /$ año. Esta escorrentía corre por las 79 cuencas hidrográficas y las 137 subcuencas que hay en el país, que están organizadas para fines de planificación en 31 sistemas hídricos. El total de estas cuencas está distribuido en dos vertientes que nacen en la Sierra Ecuatoriana y que se distribuyen hacia ambos lados de la misma. Hacia el lado occidental, la vertiente del Pacífico distribuye 72 cuencas, y hacia oriente, la vertiente del Amazonas posee 7 cuencas que ocupan $131.726 \mathrm{~km}^{2}$ del territorio nacional. 


\section{DATOS}

A partir de la base de datos de caudales diarios proporcionados por el Instituto Nacional de Meteorología e Hidrología de Ecuador (INAMHI), se llevó a cabo un control de calidad, identificándose 26 estaciones que presentaban mayor información continua desde el año 1964 a 2014. La Figura 1 muestra la ubicación de las 26 series de caudal usadas en este estudio, que se localizan sobre 25 ríos diferentes de Ecuador. Los registros son principalmente flujos naturales.
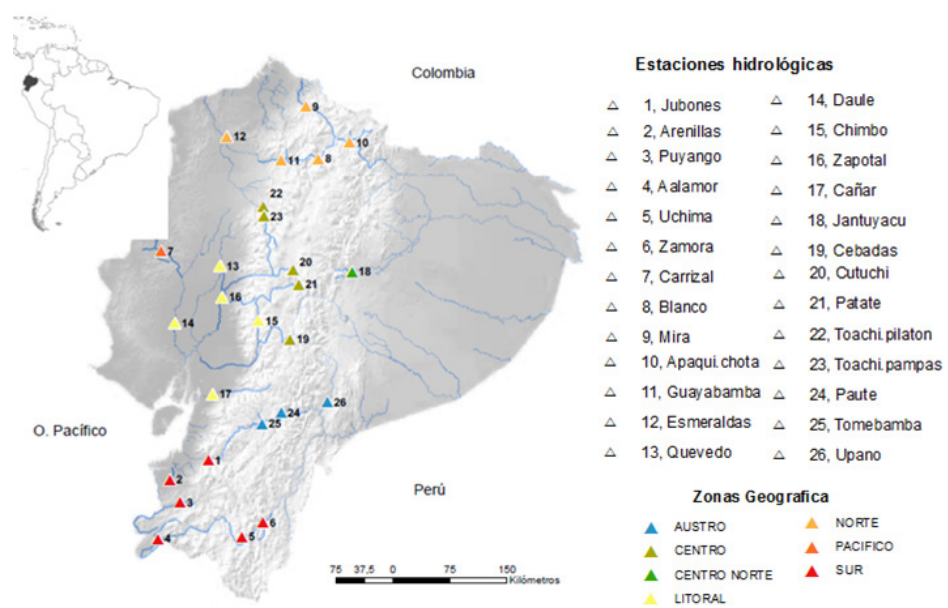

Fig. 1: Mapa de ubicación de las estaciones de caudal en Ecuador. Fuente: Instituto Nacional de Meteorología e Hidrología.

A partir de los datos de caudal diario se obtuvo el promedio mensual para cada estación. Las razones para considerar los datos mensuales de caudales son los siguientes: (1) la evaluación de la cantidad disponible de agua en una cuenca y, por lo tanto, su gestión para un suministro eficiente, se hace generalmente durante períodos de tiempo más largos, como meses y años, en lugar de hacerlo a escala diaria; y (2) las series mensuales y anuales de caudales están más relacionadas con el clima a largo plazo en comparación con las series diarias y, por lo tanto, la dinámica de flujo fluvial a estas escalas puede ser utilizada para establecer conexiones entre el clima y el caudal.

Por medio del software Amelia II (Honaker et al., 2011), que trabaja con la imputación múltiple a partir del algoritmo EM (expectation maximization), método interactivo que busca la estimación por medio de modelos estadísticos y que está diseñado específicamente para el relleno de datos de series temporales, se han rellenado los huecos de los datos en base mensual, como se muestra en la Tabla 1.

La Figura 2 presenta el ciclo anual del caudal para cuatro estaciones seleccionadas. A lo largo del año, se han detectado dos tipos distintos de distribuciones anuales de caudal. Así, existen ríos como el Quevedo y el Toachi Pilaton, que durante el primer semestre del año (de enero a junio) presentan valores altos del caudal y en el siguiente semestre (de julio a diciembre) valores más bajos, asociados a los caudales 
de las estaciones húmeda y seca, respectivamente. Este es el comportamiento más común en las estaciones analizadas. Por otro lado, ríos como el Cebadas y el Juntuyacu muestran una distribución del caudal más homogénea durante todo el año, excepto para los meses de junio, julio y agosto que viene a ser el trimestre con valores más altos de caudal.

\begin{tabular}{|c|c|c|c|c|c|c|c|c|}
\hline Estación & Lat. & Long. & $\begin{array}{c}\text { huecos } \\
(\%)\end{array}$ & $\begin{array}{c}\text { caudal } \\
\left(\mathrm{m}^{3} / \mathrm{s}\right)\end{array}$ & $\begin{array}{l}\text { caudal- } \\
\text { min } \\
\left(\mathrm{m}^{3} / \mathrm{s}\right)\end{array}$ & $\begin{array}{c}\text { caudal- } \\
\text { max } \\
\left(\mathrm{m}^{3} / \mathbf{s}\right)\end{array}$ & Cuenca & Zona \\
\hline Jatunyacu & $-1,09$ & $-77,91$ & 24 & 295,06 & 31,21 & 1432,98 & Napo & Amazonia \\
\hline Paute* & $-2,76$ & $-78,74$ & 25 & 54,35 & 0,23 & 193,66 & Santiago & Austro \\
\hline Tomebamba & $-2,9$ & $-78,97$ & 20 & 17,84 & 0,18 & 82,71 & Santiago & Austro \\
\hline Upano* & $-2,64$ & $-78,2$ & 31 & 341,4 & 3,82 & 852,29 & Santiago & Austro \\
\hline Toachi,Pampas & $-0,43$ & $-78,95$ & 13 & 21,13 & 0,34 & 82,25 & Esmeraldas & Centro \\
\hline Toachi,Pilaton & $-0,32$ & $-78,95$ & 18 & 44,25 & 0,62 & 166,45 & Esmeraldas & Centro \\
\hline Cebadas* & $-1,89$ & $-78,64$ & 6 & 20,98 & 0,96 & 116,9 & Pastaza & Centro \\
\hline Cutuchi & $-1,07$ & $-78,6$ & 8 & 10,79 & 0,13 & 40,9 & Pastaza & Centro \\
\hline Patate* & $-1,24$ & $-78,53$ & 35 & 27,08 & 0,37 & 85,32 & Pastaza & Centro \\
\hline Cañar & $-2,54$ & $-79,55$ & 24 & 57,67 & 0,65 & 317,55 & Cañar & Litoral \\
\hline Quevedo & $-1,02$ & $-79,46$ & 9 & 208,44 & 0,04 & 1109,28 & Guayas & Litoral \\
\hline Daule & $-1,7$ & $-80,00$ & 3 & 291,01 & 0 & 1910,76 & Guayas & Litoral \\
\hline Zapotal & $-1,39$ & $-79,44$ & 8 & 144,49 & 0,57 & 766,38 & Guayas & Litoral \\
\hline Chimbo & $-1,67$ & $-79,01$ & 16 & 6,02 & 0,15 & 41,09 & Guayas & Litoral \\
\hline Guallabamba & 0,24 & $-78,75$ & 24 & 135,16 & 1,56 & 715,99 & Esmeraldas & Norte \\
\hline Mira & 0,87 & $-78,45$ & 31 & 146,57 & 26,04 & 405,93 & Mira & Norte \\
\hline Blanco & 0,25 & $-78,3$ & 17 & 0,83 & 0 & 15,5 & Mira & Norte \\
\hline Apaqui,Chota* & 0,44 & $-77,94$ & 21 & 9,19 & 0,02 & 46,85 & Mira & Norte \\
\hline Carrizal & $-0,85$ & $-80,16$ & 23 & 14,7 & 0,01 & 350,77 & Chone & Pacifico \\
\hline Esmeraldas & 0,51 & $-79,38$ & 23 & 883,33 & 4,38 & 2887,34 & Esmeraldas & Pacifico \\
\hline Arenilla & $-3,56$ & $-80,06$ & 15 & 7,51 & 0,17 & 67,15 & Arenilla & Sur \\
\hline Uchima & $-4,23$ & $-79,20$ & 25 & 2,63 & 0,11 & 10,66 & Chira & Sur \\
\hline Alamor & $-4,26$ & $-80,20$ & 6 & 7,54 & 0 & 95,54 & Chira & Sur \\
\hline Jubones & $-3,32$ & $-79,6$ & 8 & 47,37 & 2,7 & 235,62 & Jubones & Sur \\
\hline Puyango & $-3,83$ & $-79,93$ & 12 & 26,18 & 1,1 & 166,42 & Puyango & Sur \\
\hline Zamora* & $-4,06$ & $-78,95$ & 33 & 81,41 & 1,63 & 311,11 & Santiago & Sur \\
\hline
\end{tabular}

Tabla1: Características de las 26 estaciones hidrológicas seleccionadas para el estudio. Las estaciones que muestran mayor valor en los meses de junio, julio y agosto están marcadas $\operatorname{con}(*)$ 

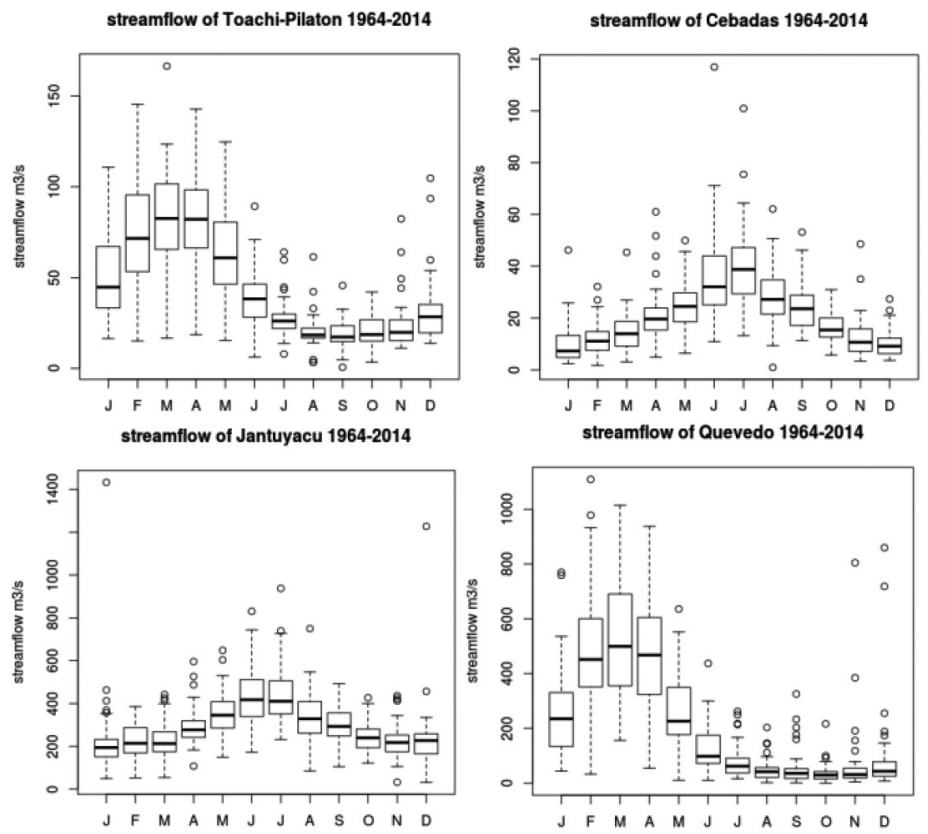

Fig. 2: Ciclo anual del caudal para cuatro estaciones seleccionadas.

\section{METODOLOGÍA}

Existen muchos métodos paramétricos y no paramétricos para la detección de tendencias aplicados a los registros hidrológicos (Kundzewicz y Robson, 2004). En general, muchos hidrólogos prefieren los métodos no paramétricos, evitando la necesidad de conocer la función de distribución de los registros hidrológicos disponibles. Por ello, en este estudio, se ha optado por el uso de métodos no paramétricos. Se ha utilizado la prueba de Mann-Kendall (Kendall, 1975) para la detección de tendencias significativas, y el método de la pendiente de Sen (Sen, 1968).para estimar la magnitud de las tendencias.

El test de Mann-Kendall (Kendall, 1975) es un test no paramétrico que evalúa el comportamiento monótono de una serie de datos. Al ser no paramétrico puede ser aplicado sin tener en cuenta la distribución estadística de los datos. Además permite detectar tendencias no necesariamente lineales. En este trabajo, se consideran tendencias muy significativas aquellas que lo sean al nivel de confianza del 95\% $(p<0.05)$, y significativas aquellas que lo sean al 90\% $(p<0.1)$.

Por otro lado, como indicador de la magnitud de las tendencias se ha usado el valor obtenido de la pendiente de Sen. El método de Sen es un método no paramétrico robusto que cuantifica la tendencia como cambio por unidad de tiempo en una serie y tiene ciertas ventajas como el hecho de permitir valores faltantes o que la muestra no se debe ajustar a una distribución en particular. Para hacer comparables los resultados 
de las tendencias entre las distintas localidades analizadas, estas tendencias se expresan en porcentaje respecto al valor medio del índice.

Adicionalmente se ha usado el coeficiente de correlación de Spearman (Kendall, 1975) para analizar la relación entre diversos índices asociados al fenómeno ENSO y los caudales estacionales.

\section{RESULTADOS}

\subsection{Variabilidad espacial de las tendencias del caudal}

Se analizan a continuación las tendencias obtenidas para cada uno de los meses de las estaciones seleccionadas (Tabla 1). La Figura 3 muestra los resultados para los meses correspondientes al primer semestre del año (estación húmeda), mientras que la Figura 4 muestra los valores de tendencias calculados para los meses correspondientes al segundo semestre del año (estación seca).
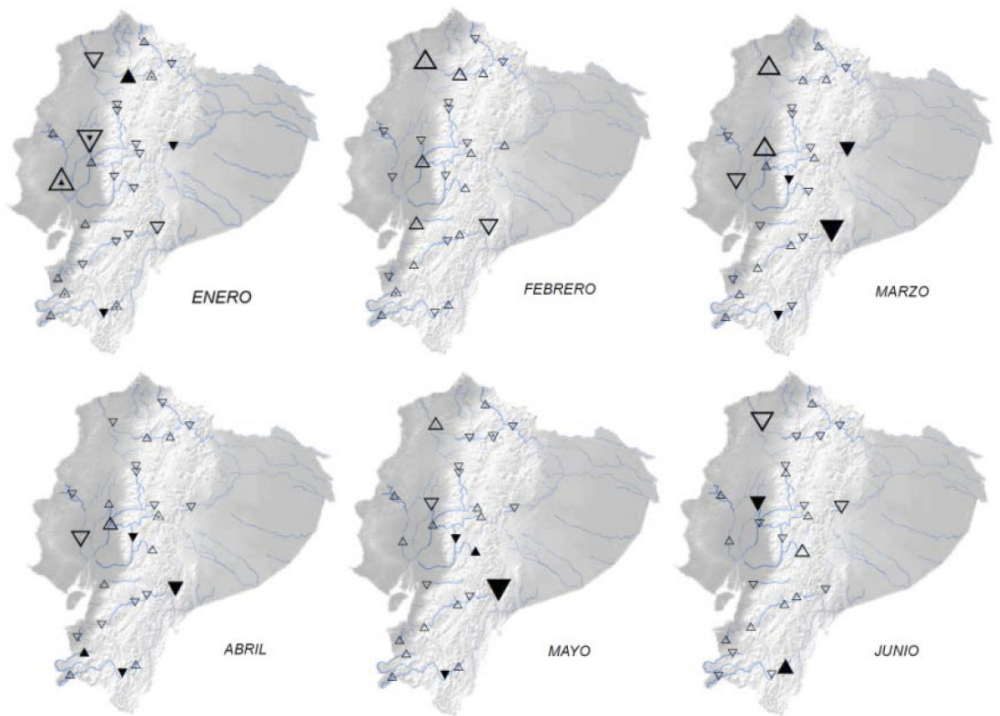

Fig. 3: Tendencias mensuales del caudal (\%) para los meses correspondientes a la estación húmeda del año. Triángulos hacia arriba o abajo indican tendencias positivas o negativas, respectivamente; rellenos de negro indican tendencias significativas al nivel de confianza del 95\%, con un triángulo menor dentro, al nivel del 90\%, y sin rellenar no significativas al nivel del 90\%. El tamaño del triángulo también indica si la tendencia relativa es alta (mayor que

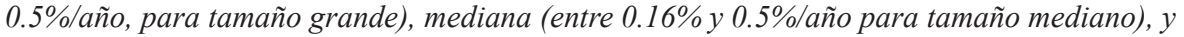
baja (menor o igual que $0.16 \% / a n ̃ o)$.

De manera general, en la Figura 3 se observan tendencias con un nivel de significación menor del $90 \%$ en la mayor parte del área de estudio, salvo algunas excepciones. Enero muestra tendencias negativas del caudal principalmente en la zona centro, austro y la Amazonia. Las estaciones de Juntuyacu, Guayabamba y Uchima son las que muestran valores significativos al 95\%. El mes de febrero parece ser un mes de transición, experimentando tendencias de signo contrario en la mayoría de las 
regiones pero con valores no significativos. En marzo los cambio que se manifestaron en febrero se mantienen pero más significativos. También en la zona del litoral se observa un incremento de la tendencia más significativa. Los meses de abril y mayo muestran un comportamiento muy similar, manteniendo en su mayoría las mismas tendencias observadas en marzo. En junio empiezan a notarse cambios de tendencia principalmente en la zona central y mientras la zona litoral, norte y sur mantienen una tendencia similar a los meses pasados.

La Figura 4 muestra los resultados para los valores de tendencias del caudal calculados para los meses correspondientes al segundo semestre del año. Aparecen cambios significativos en el comportamiento de las tendencias comparado con el primer semestre del año. Llama la atención que los meses de junio (Fig. 3) y julio (Fig. 4) mantienen cierta homogeneidad con ligeros cambios de tendencia.
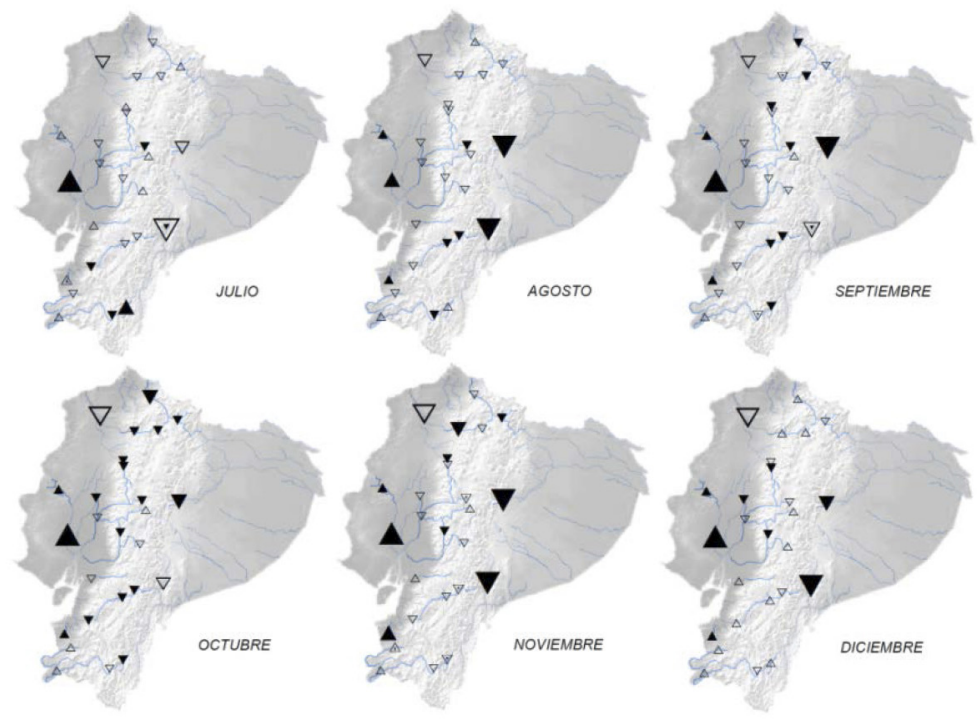

Fig. 4: Como en la Fig. 3 para los meses correspondientes a la estación seca del año.

Podría establecer el mes de julio como un periodo de transición que da lugar a tendencias significativas más claras durante agosto. Los meses de agosto a diciembre son los que muestran mayor cantidad de estaciones con tendencias significativas al 95\%, especialmente octubre, que muestra en la mayoría de los casos tendencias negativas, generalizadas en las regiones del norte, litoral y austro. En noviembre se mantienen en su mayoría los signos de tendencias presentados en octubre, aunque baja la significación, y en diciembre se empiezan a observar ligeros cambios en el comportamiento de las tendencias, principalmente en la zona norte.

\subsection{Correlación entre tendencia de caudal y ENSO}

En este apartado se analiza la relación del caudal y tres índices representativos del fenómeno de El Niño para distintos trimestres (estaciones) del año, a partir del 
análisis de la correlación de Spearman. Se ha seguido una agrupación por meses igual que la usada por Córdoba-Machado et al. (2015) al analizar la relación entre la precipitación en Colombia y los índices del El Niño 3.4 y El Niño Modoki. Además de estos dos índices, se analiza también El Niño 1+2, ya que este índice se calcula a partir de las anomalías de la temperatura de la superficie del mar en el área del Pacífico contigua a las costas de Ecuador, y porque algunos autores han mostrado la influencia de este índice en la sequía en Ecuador (Vicente-Serrano et al., 2016).

La Figura 5 muestra los valores de la correlación de Spearman en cada trimestre del año entre el caudal y el índice asociado a El Niño Modoki. Se observa que el caudal de las estaciones ubicadas en la parte más occidental del país, incluyendo la zona andina más occidental, se correlaciona negativamente con El Niño Modoki principalmente en los dos primeros trimestres del año con una significancia en su mayoría del 95\%.Los valores más marcados de esta correlaciones $(\mathrm{r}<-0.5)$ se encuentran en la zona más septentrional, sobre los Andes y en algunas estaciones del sur para la estación de DEF. Estas correlaciones negativas disminuyen parcialmente para el trimestre JJA, siendo significativas al nivel de confianza del $95 \%$ en la zona centro y en la zona costera de la región sur. Para la estación SON, de nuevo las correlaciones son negativas, pero con niveles de significación menores, de tal forma que se encuentran valores significativos por encima del $90 \%$ para estaciones ubicadas sobre los Andes.
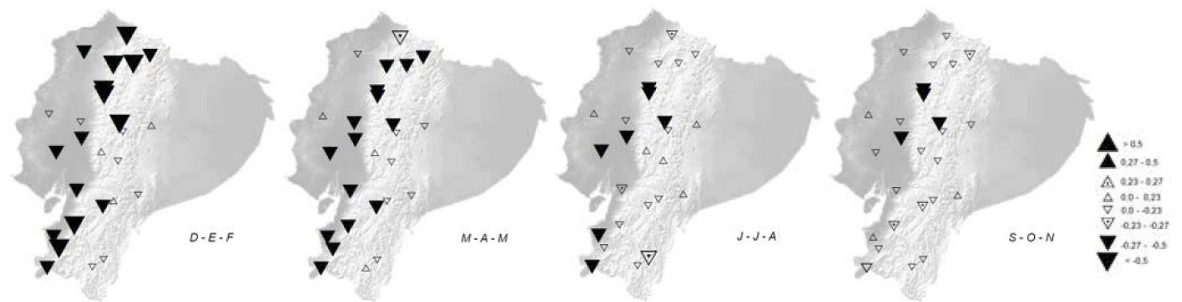

Fig. 5: Correlación entre el caudal estacional y el índice del Niño Modoki para los cuatro trimestres considerados. El significado de los símbolos es como en la Fig. 3., siendo el tamaño del triángulo indicativo del valor de $R$ (mayor que 0.5 en valor absoluto para tamaño grande, y medio entre 0.27 y 0.5 ).

La Figura 6 muestra los valores de correlación de Spearman para los caudales trimestrales y los índices estacionales de El Niño1+2. En general, las correlaciones son menos uniformes en signo y menos significativas. Así, para la zona litoral y sur hay una prevalencia de las correlaciones positivas, significativas al 95\% sobre todo en MAM, y en menor medida en JJA. Por otro lado, la mitad norte de los Andes presenta una prevalencia de correlaciones negativas, significativas por encima del $90 \%$ para DEF. Sólo la estación más al norte sobre los Andes mantiene correlaciones negativas significativas (al 95\%) en las estaciones MAM y JJA.

Por último, la Figura 7 muestra las correlaciones entre el caudal trimestral o estacional y el índice coetáneo asociado a EL Niño 3.4. Como en el caso anterior, las correlaciones son positivas en el área litoral, aunque no significativas. Para el resto del país predominan las correlaciones negativas, significativas al nivel de confianza del $95 \%$ para la mitad norte de los Andes en los tres primeros trimestres considerados. 
Más al sur, algunas estaciones también muestran correlaciones significativas, al menos al 90\%, en estas tres estaciones del año. Para SON, las correlaciones son positivas en la zona litoral y Pacífico, pero no significativas, y negativas en el resto del país, pero sólo significativas en dos estaciones sobre los Andes.
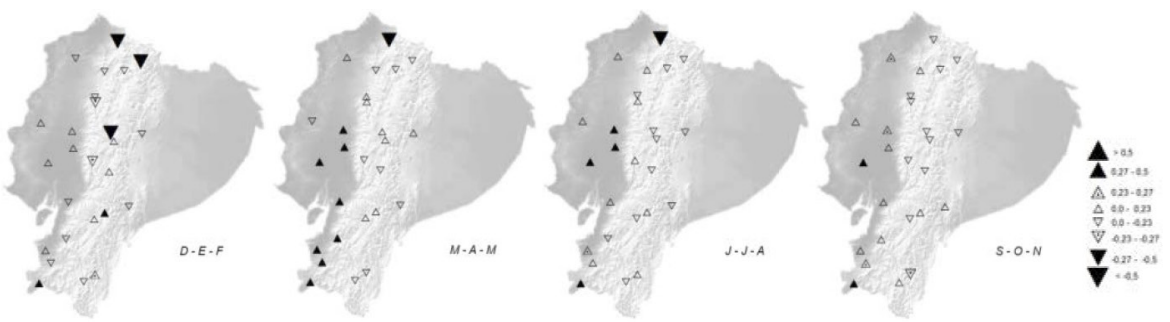

Fig. 6: Como la Figura 5 para el índice Niño 1+2.
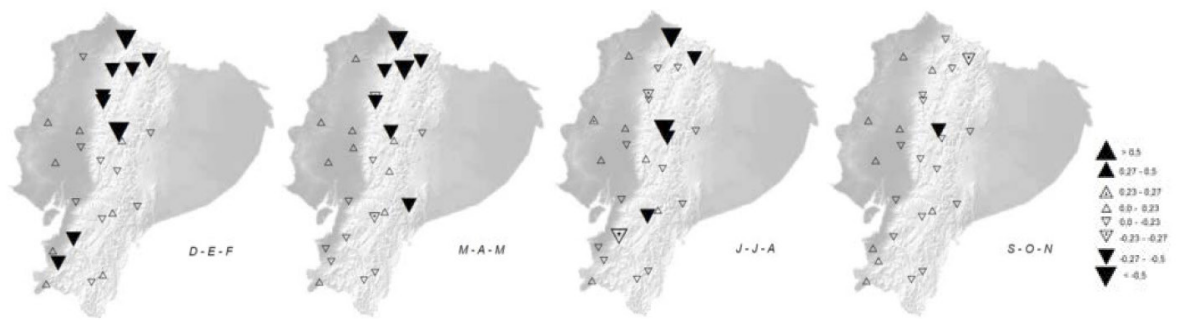

Fig. 7: Como la Figura 5 para el índice Niño 3.4.

Así pues, pueden observarse diferencias claras en el comportamiento del caudal respecto a los diversos índices del fenómeno ENSO considerado. En general, las relaciones encontrada son más significativas para la estación del DEF (cuando el fenómeno ENSO suele alcanzar su etapa madura), debilitándose a lo largo del año, del tal forma que durante SON la posible relación del caudal con el ENSO es muy débil.

\section{CONCLUSIONES}

El presente trabajo analiza las tendencias de los caudales mensuales de los ríos de Ecuador en el periodo 1964-2014, con el fin de evidenciar cambios significativos del mismo durante las estaciones húmedas y secas del año. Además se evalúa la relación existente entre diversos índices asociados al fenómeno ENSO y el caudal en base estacional siguiendo la agrupación estacional de otros trabajos para regiones vecinas (Córdoba-Machado et al., 2015)

Los principales resultados extraídos de este estudio son los siguientes:

- El ciclo anual del caudal permite establecer dos tipos de comportamiento. La mayoría de las estaciones consideradas presentan un primer semestre que abarca los meses de enero a junio (estación húmeda) y un segundo semestre que abarca de julio a diciembre (estación seca). Un conjunto más reducido de estaciones 
muestra un ciclo anual menos marcado, con valores máximos del mismo de julio a septiembre.

- Este comportamiento del caudal a lo largo del año está relacionado con la ubicación geográfica de las estaciones. Las zonas centro, sur, austro y Amazonia presentan el ciclo más homogéneo, mientras las estaciones que se encuentran más cercanas a la costa, es decir, la zona litoral sur o Pacífico muestran un comportamiento diferenciado para las estaciones húmedas y secas.

- En el primer semestre, en la mayoría de las zonas de estudio, las tendencias son predominantemente negativas y no significativas. Sin embargo, en el segundo semestre del año existen cambios significativos que muestran tendencias negativas del caudal, significativas al nivel de confianza del 95\%, en una gran parte del país. Sólo algunas estaciones situadas en la zona litoral y Pacífico del país muestran tendencias crecientes significativas durante estos meses del año.

- Para todos los índices ENSO analizados, es durante los meses de DEF cuando se presenta un mayor número de estaciones con correlaciones significativas, seguido del trimestre MAM. Por tanto el impacto de ENSO parece ser mayor durante la época húmeda en Ecuador.

- En general, el índice del ENSO que presenta un mayor número de estaciones con correlaciones significativas (negativas) es El Niño Modoki y en menor número El Niño 3+4. Sólo para el índice El Niño 1+2 se encuentran correlaciones significativas positivas en estaciones de las regiones litoral y Pacífico para la estación de MAM y en menor medida para JJA.

- Estos resultados son acordes con las relaciones encontradas entre los índices Niño3.4 y Niño 1+2 y los patrones de sequía (Vicente-Serrano et al., 2016).

\section{AGRADECIMIENTOS}

Este trabajo ha sido financiado por los proyectos P11-RNM-7941 (Junta de Andalucía-España) and CGL2013-48539-R (MINECO-España, FEDER).

\section{REFERENCIAS}

Buytaert, W., Celleri, R., Willems, P., Bièvre, B.D., Wyseure, G. (2006) Spatial and temporal rainfall variability in mountainous areas: A case study from the south Ecuadorian Andes. Journal of Hydrology 329: 413-421

Córdoba-Machado, S., Palomino-Lemus, R., Gámiz-Fortis, S., Castro-Díez, Y., Esteban-Parra, M.J. (2015) Assessing the impact of El Niño Modoki on seasonal precipitation in Colombia. Globaland Planetary Change 124:241-261.

Honaker, J., G. King, G. and Blackwell, M. (2011). Amelia II: A Program for Missing Data, Journal of Statistical Software 45, http://www.jstatsoft.org/v45/i07/

Jayawardena, A.W. and Lai, F. (1994). Analysis and prediction of chaos in rainfall and stream flow time series.Journal of Hydrology153(1-4):23-52.

Kendall, M.G. (1975). Rank Correlation Methods. Charles Griffin Book, London. 
Kundzewicz, Z. W., Robson, A. J. (2004) Change detection in river flow recordsreview of methodology. Hydrological Science Journal, 49(1), 7-19.

Sen, P.K. (1968). Estimates of the regression coefficient based on Kendall's tau. Journal of the American Statistical Associations 63 (324), 1379-1389.

Vicente-Serrano, S.M., Aguilar, E., Martínez, R., Martín-Hernández, N., AzorinMolina, C., Sanchez-Lorenzo, A., El Kenawy, A., Tomás-Burguera, M., MoranTejeda, E., López-Moreno, J.I., Revuelto, J., Beguería, S., Nieto, J.J., Drumond, A., Gimeno, L., Nieto, R. (2016). The complex influence of ENSO on droughts in Ecuador. Climate Dynamics, DOI 10.1007/s00382-016-3082-y.

Vuille, M., Bradley, R.S., Keimig, F. (2000). Climate variability in the Andes of Ecuador and its relation to tropical Pacific and Atlantic sea surface temperatures anomalies. Journal of Climate 13, 2520-2535. 